PROCEEDINGS OF THE

AMERICAN MATHEMATICAL SOCIETY

Volume 125, Number 5, May 1997, Pages 1523-1530

S 0002-9939(97)03702-7

\title{
LESS SATURATED IDEALS
}

\author{
MOTI GITIK AND SAHARON SHELAH
}

(Communicated by Andreas R. Blass)

\begin{abstract}
We prove the following:
(1) If $\kappa$ is weakly inaccessible then $N S_{\kappa}$ is not $\kappa^{+}$-saturated.

(2) If $\kappa$ is weakly inaccessible and $\theta<\kappa$ is regular then $N S_{\kappa}^{\theta}$ is not $\kappa^{+}$saturated.

(3) If $\kappa$ is singular then $N S_{\kappa^{+}}^{c f \kappa}$ is not $\kappa^{++}$-saturated.

Combining this with previous results of Shelah, one obtains the following:

(A) If $\kappa>\aleph_{1}$ then $N S_{\kappa}$ is not $\kappa^{+}$-saturated.

(B) If $\theta^{+}<\kappa$ then $N S_{\kappa}^{\theta}$ is not $\kappa^{+}$-saturated.
\end{abstract}

\section{INTRODUCTION}

By a classical result of $R$. Solovay [So] every stationary subset $S$ of a regular cardinal $\kappa$ can be split into $\kappa$ disjoint stationary subsets. Or, in other terminology, $N S_{\kappa}\lceil S$, the nonstationary ideal over $\kappa$ restricted to a stationary subset $S$ of $\kappa$, is not $\kappa$-saturated.

A natural question is if it is possible to replace $\kappa$-saturated ness by $\kappa^{+}$-saturatedness, i.e. instead of $\kappa$ completely disjoint stationary subsets to have $\kappa^{+}$with pairwise nonstationary intersection. K. Namba [Na] proved that $N S_{\kappa}$ cannot be $\kappa^{+}$-saturated over a measurable $\kappa$. J. Baumgartner, A. Taylor and S. Wagon [Ba-Ta-Wa] improved this to a greatly Mahlo. S. Shelah [Sh1] showed that $N S_{\kappa^{+}} \uparrow$ $S$ is not $\kappa^{++}$-saturated if for some regular $\theta<\kappa, \theta \neq \operatorname{cf} \kappa, S \cap\left\{\alpha<\kappa^{+} \mid c f \alpha=\theta\right\}$ is stationary. Actually a much more general result is proved there. For $\kappa=\aleph_{1}$, it is possible to have $N S_{\kappa} \kappa^{+}$-saturated, as was shown by J. Steel and R. Van Wesep [St-V] from $A D_{R}$, by H. Woodin [Wo1] from $A D$, by M. Foreman, M. Magidor and S. Shelah [Fo-Ma-Sh] from a supercompact and by S. Shelah [Sh6] from a Woodin cardinal. Also $N S_{\kappa^{+}}\left\lceil S\right.$ can be $\kappa^{++}$-saturated for a regular $\kappa$ and $S \subseteq\left\{\alpha<\kappa^{+} \mid \operatorname{cf} \alpha=\kappa\right\}$, as was shown by T. Jech and H. Woodin [Je-Wo] building on K. Kunen's $[\mathrm{Ku}]$ construction of a saturated ideal. If $\kappa$ is singular, then, by M. Foreman [Fo] and T. Jech, H. Woodin [Je-Wo], $N S_{\kappa^{+}} \uparrow S$ can be $\kappa^{++}$-saturated for $S \subseteq\left\{\alpha<\kappa^{+} \mid \operatorname{cf} \alpha=\operatorname{cf} \kappa\right\}$.

If $\kappa$ is an inaccessible then T. Jech and H. Woodin [Je-Wo] showed that $N S_{\kappa}\left\lceil\right.$ Regulars can be $\kappa^{+}$-saturated, and in [Gi2] was shown the consistency of

Received by the editors March 1, 1995 and, in revised form, November 20, 1995.

1991 Mathematics Subject Classification. Primary 03E35, 03E40, 04A20.

The second author was partially supported by the Basic Research Fund, Israel Academy of Sciences.

This is number 577 in the cumulative list of the second author's publications.

(C)1997 American Mathematical Society 
$N S_{\kappa}\left\lceil S \kappa^{+}\right.$-saturated for $S \subseteq \kappa$ such that $S \cap\{\alpha<\kappa \mid$ cf $\alpha=\theta\}$ is stationary for every regular $\theta<\kappa$.

The purpose of the present paper is to show that $N S_{\kappa}$ and $N S_{\kappa}^{\theta}=N S_{\kappa} \uparrow\{\alpha \mid$ cf $\alpha=\theta$ \} cannot be $\kappa^{+}$-saturated where $\kappa$ is weakly inaccessible and $\theta<\kappa$. And for singular $\kappa, N S_{\kappa^{+}}^{\mathrm{cf} \kappa}$ cannot be $\kappa^{++}$-saturated.

Combining this with the previous result of Shelah [Sh1], one gets the following:

Theorem. Let $\kappa$ and $\theta$ be regular cardinals. Then:

(1) If $\kappa>\aleph_{1}$ then $N S_{\kappa}$ is not $\kappa^{+}$-saturated.

(2) If $\theta^{+}<\kappa$ then $N S_{\kappa}^{\theta}$ is not $\kappa^{+}$-saturated.

The proof is based on a certain combinatorial principle which follows from saturatedness. It was considered independently by both authors. Actually they came to it from different directions. Gitik was dealing with indiscernibles of the Mitchell Covering Lemma and showed it first under $\neg \exists \kappa o(\kappa)=\kappa^{++}$in [Gi3], and then removed this assumption in [Gi4]. Shelah [Sh2, Sh3, Dz-Sh] went through his club guessing machinery and eventually obtained a better result not appealing to some GCH assumptions used in [Gi4]. He also realized that this leads to inconsistency of saturatedness of $N S_{\kappa}^{\theta}$ over small inaccessibles. A minor change in this argument by Gitik led to the full result for inaccessibles. Shelah saw that it gives also the nonsaturatedness of $N S_{\kappa^{+}}^{\text {cf }}$ for a singular $\kappa$.

We are grateful to A. Blass, M. Kojman, P. Matet and the referee of the paper for their remarks and corrections.

\section{MAin RESUlt}

Let $\kappa$ be a regular uncountable cardinal. We denote by $N S_{\kappa}$ the nonstationary ideal over $\kappa$. For a set $S \subseteq \kappa$ let $N S_{\kappa} \uparrow S$ denote the collection of all subsets of $\kappa$ having a nonstationary intersection with $S$. For a regular $\theta<\kappa$ let

$$
S_{\kappa}^{\theta}=\{\alpha<\kappa \mid \operatorname{cf} \alpha=\theta\} .
$$

We denote $N S_{\kappa} \uparrow S_{\kappa}^{\theta}$ simply by $N S_{\kappa}^{\theta}$.

An ideal $I$ over $\kappa$ is called $\lambda$-saturated if there is no sequence $\left\langle A_{\alpha} \mid \alpha<\lambda\right\rangle$ so that

(a) $A_{\alpha} \subseteq \kappa$,

(b) $A_{\alpha} \notin I$,

(c) $A_{\alpha} \cap A_{\beta} \in I$ whenever $\alpha \neq \beta$.

Our main objective will be the non- $\kappa^{+}$-saturatedness of $N S_{\kappa}^{\theta}$. So from here on, by a saturated ideal over a cardinal $\lambda$ we will mean a $\lambda^{+}$-saturated ideal.

We will prove the following:

Theorem 1. (1) If $\kappa$ is weakly inaccessible then $N S_{\kappa}$ cannot be saturated.

(2) If $\kappa$ is weakly inaccessible and $\theta<\kappa$ is regular then $N S_{\kappa}^{\theta}$ cannot be saturated.

(3) If $\kappa$ is a singular cardinal then $N S_{\kappa^{+}}^{\text {cf } \kappa}$ cannot be saturated.

Actually, the proof will show the Theorem of the Introduction for every regular $\kappa>\aleph_{2}$.

The proof will consist of two parts. The first will introduce a combinatorial principle and show its inconsistency. The second will show that the saturatedness of $N S_{\kappa}^{\theta}$ implies the principle. 
Definition 2. For regular cardinals $\kappa, \theta$ with $\kappa>\aleph_{2}$ and $\kappa>\theta^{+}$let $\diamond_{\text {club }}^{*}(\kappa, \theta)$ denote the following: there exists a sequence $\left\langle S_{\alpha} \mid \alpha \in S_{\kappa}^{\theta}\right\rangle$ so that for every $\alpha \in S_{\kappa}^{\theta}$

(1) $S_{\alpha} \subseteq \alpha$,

(2) $\sup S_{\alpha}=\alpha$,

(3) $\left|S_{\alpha}\right|=\theta$

(4) if $\alpha$ is a limit of ordinals of cofinality $>\theta$ (and $>\aleph_{1}$ if $\theta=\aleph_{0}$ ), then for every $\beta \in S_{\alpha} \operatorname{cf} \beta>\theta$ and if $\theta=\aleph_{0}$ then $\operatorname{cf} \beta>\aleph_{1}$,

(5) for every club $C \subseteq \kappa$ the set

$$
\left\{\alpha \in S_{\kappa}^{\theta} \mid \exists \beta<\alpha C \supseteq S_{\alpha} \backslash \beta\right\}
$$

contains a club intersected with $S_{\kappa}^{\theta}$.

Notice that all but nonstationary many $\alpha$ 's in $S_{\kappa}^{\theta}$ are limits of ordinals of cofinality $>\theta$.

This is a strengthening of Shelah's club guessing principles; see [Sh2, Sh3]. It turns out that it is too strong. Namely:

Lemma 3. $Z F C \vdash \neg \diamond_{\text {club }}^{*}(\kappa, \theta)$.

Proof. Suppose otherwise. Let $\left\langle S_{\alpha} \mid \alpha \in S_{\kappa}^{\theta}\right\rangle$ be a sequence witnessing $\diamond_{\text {club }}^{*}(\kappa, \theta)$.

Let us split the proof into two cases according to whether $\theta>\aleph_{0}$ or $\theta=\aleph_{0}$.

Case 1. $\theta>\aleph_{0}$.

We define by induction a sequence $\left\langle E_{n} \mid n<\omega\right\rangle$ of clubs of $\kappa$. Let $E_{0}$ be the set of ordinals below $\kappa$ which are limits of ordinals of cofinality $>\theta$. If $E_{n}$ is defined then consider $E_{n}^{\prime}$, the set of limit points of $E_{n}$. There exists a club $C \subseteq \kappa$ such that for every $\alpha \in C \cap S_{\kappa}^{\theta}$, $E_{n}^{\prime}$ contains a final segment of $S_{\alpha}$. Set $E_{n+1}=E_{n}^{\prime} \cap C$.

Finally let $E=\bigcap_{n<\omega} E_{n}$. Then $E$ is a club. Let $\delta=\min \left(E \cap S_{\kappa}^{\theta}\right)$. For every $n<\omega, E_{n}^{\prime}$ contains a final segment of $S_{\delta}$ since $\delta \in E_{n+1}$. But cf $\delta=\theta>\aleph_{0}$. So a final segment of $S_{\delta}$ is contained in $E$. Pick some $\beta \in E \cap S_{\delta}$. By Definition 2(4), $\operatorname{cf} \beta>\theta$. Since $E_{n}^{\prime} \supseteq E_{n+1}, \beta \in E_{n}^{\prime}$ for every $n<\omega$. So it is a limit point of $E_{n}$. Hence $E_{n} \cap \beta$ is a club of $\beta$, for every $n<\omega$. Since $\operatorname{cf} \beta>\aleph_{0}$, also $E \cap \beta$ is a club of $\beta$. But $\operatorname{cf} \beta>\theta$. Hence there is some $\gamma \in E \cap \beta$ of cofinality $\theta$, which contradicts the minimality of $\delta$.

Case 2. $\theta=\aleph_{0}$

We define a decreasing sequence of clubs as above of length $\aleph_{1}$ taking intersections at countable limit stages. Let $\left\langle E_{i} \mid i<\omega_{1}\right\rangle$ be such a sequence and let $E=\bigcap_{i<\omega_{1}} E_{i}$. As in Case 1 pick $\delta$ to be $\min \left(E \cap S_{\kappa}^{\aleph_{0}}\right)$. By Definition 2(3), $\left|S_{\delta}\right|=\omega$. Let $\left\langle s_{n} \mid n<\omega\right\rangle$ be a cofinal in $\delta$ sequence in $S_{\delta}$. Since for every $i<\omega_{1}$ $E_{i+1}$ contains a final segment of $S_{\delta}$, there will be some $n^{*}<\omega$ such that for $\aleph_{1} i$ 's we have $E_{i+1} \supseteq\left\{s_{n} \mid n \geq n^{*}\right\}$. But the sequence $\left\langle E_{i} \mid i<\omega_{1}\right\rangle$ is a decreasing sequence. So for every $i<\omega_{1}, E_{i} \supseteq\left\{s_{n} \mid n \geq n^{*}\right\}$. Hence there is $\beta \in E \cap S_{\delta}$. Notice that by Definition 2(4), ef $\beta>\aleph_{1}$. Now we continue toward the contradiction as in Case 1.

Lemma 4. Suppose that $\kappa, \theta$ are regular cardinals and $\theta^{+}<\kappa$. If $N S_{\kappa}^{\theta}$ is saturated then $\diamond_{\text {club }}^{*}(\kappa, \theta)$ holds.

Remark. Both authors arrived to this statement independently and from different directions. Gitik, dealing with indiscernibles of Mitchell's Covering Lemma, showed this under $\neg\left(o(\alpha)=\alpha^{++}\right)$and $2^{\theta}<\kappa$ in [Gi3], and much later in [Gi4] realized that 
$\neg\left(o(\alpha)=\alpha^{++}\right)$is not needed. Shelah went through his club guessing principles [Sh2, Sh3] and got a better result, requiring only $\theta^{+}<\kappa$ and not $2^{\theta}<\kappa$. The proof below follows his lines.

Proof. Let $\diamond_{\text {club }}^{\prime}(S)$ for $S$ a stationary subset of $S_{\kappa}^{\theta}$ be defined as $\diamond_{\text {club }}^{*}(\kappa, \theta)$ only with $S$ replacing $S_{\kappa}^{\theta}$ and with Definition 2(5) replaced by the following:

$(5)^{\prime}$ for every club $C \subseteq \kappa$ the set

$$
\left\{\alpha \in S \mid \exists \beta<\alpha \quad C \supseteq S_{\alpha} \backslash \beta\right\}
$$

is stationary.

It was shown by Shelah [Sh2, Sh3] that $\diamond_{\text {club }}^{\prime}(S)$ and even stronger principles are true in ZFC. For the benefit of the reader we will present a proof of $\diamond_{\text {club }}^{\prime}(S)$ below. But first let us use it to complete the proof of the lemma.

Claim 2.1. For every stationary $S \subseteq S_{\kappa}^{\theta}$ and every $\diamond_{\text {club }}^{\prime}(S)$ sequence $\left\langle S_{\alpha} \mid \alpha \in S\right\rangle$ there exists a stationary $S^{*} \subseteq S$ such that for every club $C \subseteq \kappa$ the set

$$
\left\{\alpha \in S^{*} \mid \exists \beta<\alpha \quad C \supseteq S_{\alpha} \backslash \beta\right\}
$$

contains a club intersected with $S^{*}$.

Proof. Suppose otherwise. Let $S$ be a set witnessing this. We define by induction an almost (modulo $N S_{\kappa}$ ) decreasing sequence $\left\langle C_{\alpha} \mid \alpha<\kappa^{+}\right\rangle$of clubs of $\kappa$ and an almost disjoint sequence $\left\langle A_{\alpha} \mid \alpha<\kappa^{+}\right\rangle$of stationary subsets of $S$. Denote for a club $C$ by $N(C)$ the set of places where $C$ is guessed, i.e.

$$
\left\{\alpha \in S \mid \exists \beta<\alpha \quad C \supseteq S_{\alpha} \backslash \beta\right\} .
$$

Pick $C_{0}$ to be a club such that $S \backslash N\left(C_{0}\right)$ is stationary. Set $A_{0}=S \backslash N\left(C_{0}\right)$. Suppose now that $\left\langle C_{\beta} \mid \beta<\alpha\right\rangle$ and $\left\langle A_{\beta} \mid \beta<\alpha\right\rangle$ are defined. We want to define $C_{\alpha}$ and $A_{\alpha}$. First take a club $C$ which is almost contained in every $C_{\beta}$ for $\beta<\alpha$. Consider $N(C)$. Obviously $N(C)$ is almost contained in every $N\left(C_{\beta}\right)$ for $\beta<\alpha$. Let $C_{\alpha}$ be a club subset of $C$ witnessing that $N(C)$ is not good, i.e. $N(C) \backslash N\left(C_{\alpha}\right)$ is stationary. Set $A_{\alpha}=N(C) \backslash N\left(C_{\alpha}\right)$. This completes the inductive definition.

The existence of the sequence $\left\langle A_{\alpha} \mid \alpha<\kappa^{+}\right\rangle$contradicts the saturatedness of $N S_{\kappa}^{\theta} . \quad \square$ (claim)

Now using saturatedness, it is easy to glue together the sets $S^{*}$ given by Claim 2.1 and to get $\diamond_{\text {club }}^{*}(\kappa, \theta)$. Just pick a maximal pairwise almost disjoint collection of such $S^{*}$ 's. Since $N S_{\kappa}^{\theta}$ is saturated, it consists of at most $\kappa$ sets. Then make them completely disjoint using the normality of $N S_{\kappa}^{\theta}$. Finally, we put together the sequences witnessing $\diamond_{\text {club }}^{\prime}$ and obtain $\diamond_{\text {club }}^{*}(\kappa, \theta)$. This completes the proof of the lemma.

Remark. We can replace $N S_{\kappa}^{\theta}$ by any normal ideal over $\kappa$ concentrating on $S_{\kappa}^{\theta}$ in Lemma 4. Only the definition of $\diamond_{\text {club }}^{*}(\kappa, \theta)$ must be changed in the obvious way. The same proof works. We refer to [Gi4] for generalizations for ideals preserving $2^{\theta}$ and to Dzamonja, Shelah [Dz-Sh] for further generalizations in this direction.

Let us now present a proof of Shelah's club guessing principle; see [Sh2, Sh3, Br-Ma] for variations of it.

Proposition 5. Suppose that $\kappa, \theta$ are regular and $\kappa>\max \left(\theta^{+}, \aleph_{2}\right)$. Then $\diamond_{\text {club }}^{\prime}(S)$ holds for every stationary $S \subseteq S_{\kappa}^{\theta}$.

Remark. Actually, we will show that there is a sequence $\left\langle S_{\alpha} \mid \alpha \in S\right\rangle$ witnessing $\diamond_{\text {club }}^{\prime}(S)$ so that for every club $C \subseteq \kappa,\left\{\alpha \in S \mid C \supseteq S_{\alpha}\right\}$ is stationary. 
Proof. Suppose for simplicity that $\theta^{+} \geq \aleph_{2}$. Otherwise we do the same only with $\theta^{+}$replaced by $\theta^{++}$.

Suppose that for some stationary $S \subseteq S_{\kappa}^{\theta}$, $\diamond_{\text {club }}^{\prime}(S)$ fails. We shall define sequences $\left\langle C_{i} \mid i<\theta^{+}\right\rangle$of clubs of $\kappa,\left\langle T_{\alpha}^{i} \mid i<\theta^{+}, \alpha \in S\right\rangle$ of trees and $\left\langle S_{\alpha}^{i} \mid i<\theta^{+}, \alpha \in S\right\rangle$. However, first for a club $C$ with nonlimit points of cofinality $>\theta$ and an ordinal $\alpha \in S_{\kappa}^{\theta}$ which is a limit of ordinals of cofinality $>\theta$, let us define canonically a tree $T_{\alpha}(C)$. Notice that every club contains a subclub with nonlimit points of cofinality $>\theta$. Also all but nonstationary many $\alpha$ 's are limits of ordinals of cofinality $>\theta$. The first level of $T_{\alpha}(C)$ will consist of a closed cofinal in $\alpha$ sequence of order type $\theta$ so that each nonlimit point of it has cofinality $>\theta$. We pick such a sequence to be the least in some fixed well-ordering.

Now let $\eta$ be a point from the first level. We want to define the set of its immediate successors, i.e., the set $S u c_{T_{\alpha}(C)}(\eta)$. If $\eta$ is a limit point then $\operatorname{Suc}_{T_{\alpha}(C)}(\eta)=\emptyset$. Otherwise, let $\eta^{*}$ be the largest point of the first level below $\eta$, or 0 if $\eta$ is the least one. Consider $\eta^{\prime}=\sup (C \cap \eta+1)$. If $\eta^{\prime} \leq \eta^{*}$ then set $S u c_{T_{\alpha}(C)}(\eta)=\emptyset$. Suppose that $\eta^{*}<\eta^{\prime} \leq \eta$. If cf $\eta^{\prime}>\theta$ then set $S u c_{T_{\alpha}(C)}(\eta)=\left\{\eta^{\prime}\right\}$. Such $\eta^{\prime}$ will be a leaf, i.e. $S u c_{T_{\alpha}(C)}\left(\eta^{\prime}\right)=\emptyset$. If cf $\eta^{\prime} \leq \theta$, then, as above, we pick the least closed cofinal in $\eta^{\prime}$ sequence of order type of $\operatorname{cf} \eta^{\prime}$ with nonlimit points of cofinality $>\theta$ and the first element above $\eta^{*}$ if it exists. Otherwise, set $S u c_{T_{\alpha}(C)}\left(\eta^{\prime}\right)=\emptyset$. The set of immediate successors of $\eta$ will consist of this sequence. Using it, we continue to define $T_{\alpha}(C)$ above $\eta$ in the same fashion.

Obviously the tree $T_{\alpha}(C)$ so defined is well founded and of cardinality $\leq \theta$.

Now let us turn to the definition of the sequences. Let $C_{0}$ be a a club with nonlimit points of cofinality $>\theta$. For $\alpha \in S$ which is a limit of ordinals of cofinality $>\theta$ set $T_{\alpha}^{0}=T_{\alpha}\left(C_{0}\right)$ and $S_{\alpha}^{0}=$ the set of all points of all the levels of $T_{\alpha}^{0}$ which are in $C_{0}$ and have cofinality $>\theta$. Clearly, for all but nonstationarily many $\alpha$ 's in $S, S_{\alpha}^{0}$ is unbounded in $\alpha$. This will be the case for every $\alpha \in S$ which is a limit of elements of $C_{0}$ of cofinality $>\theta$.

From here on we ignore this nonstationary set. In order to have $S_{\alpha}^{0}$ defined for every $\alpha \in S$ we may set $S_{\alpha}^{0}$ to be an unbounded in $\alpha$ sequence of order type $\theta$ whenever $S_{\alpha}^{0}$ was not defined or was defined and was bounded in $\alpha$.

Now let $C_{1}$ be a club subset of $C_{0}$ with every nonlimit point of cofinality $>\theta$ witnessing the failure of $\left\langle S_{\alpha}^{0} \mid \alpha \in S\right\rangle$ to be a $\diamond_{\text {club }}^{\prime}(S)$ sequence. We define $T_{\alpha}^{1}$ and $S_{\alpha}^{1}$ as above with $C_{1}$ replacing $C_{0}$. Continue by induction. At limit stages $i$ we take $C_{i}$ to be a club subset of $\bigcap_{j<i} C_{j}$ with nonlimit points of cofinality $>\theta$.

Finally, let $D=\bigcap_{i<\theta^{+}} C_{i}$. Then $D$ is a club since $\kappa>\theta^{+}$. Let $\alpha \in D \cap S$ be so that elements of $D$ of cofinality $>\theta$ are unbounded in it. Let us show that the trees $T_{\alpha}\left(C_{i}\right)\left(i<\theta^{+}\right)$must stabilize, i.e., starting with some $i_{\alpha}<\theta^{+}, T_{\alpha}\left(C_{i}\right)=T_{\alpha}\left(C_{j}\right)$. Otherwise, pick some $\eta_{0} \in \operatorname{Lev}_{1}\left(T_{\alpha}^{0}\right)$ such that there is no stabilization above it.

Notice that the first level in all the trees is the same. Then $\eta_{0}$ cannot be a limit point since otherwise it will have the empty set of successors. Let $\eta_{0}^{*}$ be the largest point of the first level below $\eta_{0}$, or 0 if $\eta_{0}$ is the least one. Since there is no stabilization above $\eta_{0}$ in the trees and the $C_{i}$ 's are decreasing, the sequence $\left\langle\eta_{0 i} \mid i<\theta^{+}\right\rangle$where $\eta_{0 i}=\sup \left(C_{i} \cap\left(\eta_{0}+1\right)\right)$ will be a nonincreasing sequence of ordinals inside the interval $\left(\eta_{0}^{*}, \eta_{0}\right]$. Hence it is eventually constant. So there is some $\eta_{1} \in\left(\eta_{0}^{*}, \eta_{0}\right)$, cf $\eta_{1} \leq \theta$, such that $\eta_{0 i}=\eta_{1}$ starting with some $i(1)<\theta^{+}$. By the definition of the trees the set of immediate successors of $\eta_{1}$ will be the same in every $T_{\alpha}\left(C_{i}\right)(i \geq i(1))$. Pick $\eta_{2}$ to be one of them with no stabilization above it. 
Deal with it as we did with $\eta_{0}$. We will obtain $\eta_{3}$ and $\eta_{4}$. Continue in the same fashion. This process will produce an infinite decreasing sequence of ordinals.

Hence, there is $i_{\alpha}<\theta^{+}$such that $T_{\alpha}^{i}=T_{\alpha}^{i_{\alpha}}$ for every $i \geq i_{\alpha}$. Then there are $i^{*}<\theta^{+}$and a stationary $S^{*} \subseteq S$ such that for every $\alpha \in S^{*}$ we have $i_{\alpha}=i^{*}$ and $\sup S_{\alpha}^{i^{*}}=\alpha$. But this contradicts the choice of $C_{i^{*}+1}$.

\section{Some OPEN PROBLEMS ON THE NONSTATIONARY IDEAL}

The following is probably the most interesting problem left open on this subject:

Problem 1. Let $\kappa$ be a regular uncountable cardinal. Can $N S_{\kappa^{+}}^{\kappa}$ be saturated?

By Shelah [Sh1] the answer is negative if we replace $N S_{\kappa^{+}}^{\kappa}$ by $N S_{\kappa^{+}}^{\theta}$ for some $\theta<\kappa$.

Now for $\kappa=\aleph_{0}$, the models with $N S_{\aleph_{1}}$ saturated were constructed by J. Steel and R. Van Wesep [St-V], by H. Woodin [Wo1] using $A D_{R}$ and $A D$, then by M. Foreman, M. Magidor and S. Shelah [Fo-Ma-Sh] from a supercompact and by S. Shelah [Sh6] from a Woodin cardinal. By J. Steel [St] "There are a saturated ideal over $\aleph_{1}$ and a measurable" implies an inner model with a Woodin cardinal. However, the following basic question remains open:

Problem 2. Is $G C H+N S_{\aleph_{1}}$ is saturated consistent?

A very tight connection of $\neg C H$ with saturatedness of $N S_{\aleph_{1}}$ was established by H. Woodin. He showed that " $N S_{\aleph_{1}}$ is saturated and there is a measurable" implies $\neg C H$.

If we relax our assumptions and consider saturatedness of $N S_{\kappa}\lceil S$ for a stationary set $S$, then by T. Jech and H. Woodin $N S_{\kappa^{+}} \uparrow S$ can be saturated for every $\kappa$. Also $N S_{\kappa} \uparrow$ Regulars can be saturated for inaccessible $\kappa$. An almost huge is used for the first result and a measurable for the second. By [Gi2], $N S_{\kappa}\lceil S$ can be saturated over an inaccessible $\kappa$ with $S$ having a stationary intersection with every $S_{\kappa}^{\theta}$ for $\theta$ regular less than $\kappa ; o(\kappa)=\kappa$ is used for this and it is necessary. But in order to obtain the same over the first inaccessible, supercompacts are used.

Problem 3. How strong is $N S_{\kappa} \uparrow S$ saturated for the first inaccessible $\kappa$ and $S \subseteq S_{\kappa}^{\theta}, \theta<\kappa$ regular?

One can try to get simultaneously many $S$ 's for which $N S_{\kappa} \uparrow S$ is saturated. So the following is natural: we call $N S_{\kappa}$ densely saturated if for every stationary $S \subseteq \kappa$ there is $S^{*} \subseteq S$ such that $N S_{\kappa} \uparrow S^{*}$ is saturated.

Problem 4. Can $N S_{\kappa}$ be densely saturated over an inaccessible $\kappa$ ?

Further weakenings lead to notions of presaturatedness and precipitousness introduced by J. Baumgartner-A. Taylor [Ba-Ta] and T. Jech-K. Prikry [Je-Pr] respectively.

An ideal $I$ over $\kappa$ is called presaturated if forcing with it preserves $\kappa^{+}$. It is precipitous if the generic ultrapower is well-founded. Saturatedness implies presaturatedness and presaturatedness implies precipitousness. It turned out that for every $\kappa$ $N S_{\kappa}$ can be precipitous. With presaturatedness the situation is less clear. Namely, $N S_{\kappa}$ can be presaturated for $\kappa=\aleph_{1}$ or $\kappa$ an inaccessible, but by [Sh1] these are the only cases. See Jech-Magidor-Mitchell-Prikry [Je-Ma-Mi-Pr, Gi1, Gi2, Gi3, Gi4], M. Foreman, M. Magidor, S. Shelah [Fo-Ma-Sh] for the consistency results. We do not know the answer to the following

Problem 5. Can $N S_{\aleph_{2}}^{\aleph_{1}}$ be presaturated? 
Another direction is to consider $\diamond_{\kappa}$. Obviously $\diamond_{\kappa} \longrightarrow\left(N S_{\kappa}\right.$ is not saturated $)$. One can try to prove $\diamond_{\kappa}$ in $Z F C+$ instances of $G C H$, of course. Since by Foreman and Woodin [Fo-Wo] $G C H$ can fail everywhere, so can $\diamond_{\kappa}$. H. Woodin [Cu-Wo] showed the consistency of $\neg \diamond_{\kappa}$ over the first inaccessible. But $G C H$ fails on a club of $\kappa$. He started from a supercompact. R. Jensen proved that at least a measurable is needed for this.

Problem 6. Is it consistent that $\kappa$ is an inaccessible, $\neg \diamond_{\kappa}$ and $G C H$ ?

By [Sh5], it is possible to have a stationary subset $S$ of an inaccessible $\kappa$ with $\neg \diamond_{S}$ and $G C H$.

Problem 7. Is it consistent that $2^{\kappa}=\kappa^{+}$and $\neg \diamond_{S_{\kappa^{+}}^{\text {cf } \kappa}}$ for a singular $\kappa$ ? The same for $\neg \diamond_{\kappa^{+}}$?

It is known that large cardinals are needed for this; see [Sh5]. By Gregory [Gr] and [Sh4], $\diamond_{S_{\lambda}^{\kappa}}$ holds for a cardinal $\lambda=2^{\mu}=\mu^{+}$and regular $\kappa<\mu$ provided $\mu^{\kappa}=\mu$ or $\mu$ is singular, $\kappa$ differs from cf $\mu$, and for every $\delta<\mu, \delta^{\kappa}<\mu$. This was continued in [Sh9]. Thus it was shown that for $\kappa$ above $\beth_{\mu}, \diamond_{\kappa^{+}}$is equivalent to $2^{\kappa}=\kappa^{+}$. Also see [Sh5] and Dzamonja, Shelah [Dz-Sh2] for related results. On the other hand, Shelah showed that it is consistent that $G C H$ and $\neg \diamond_{S_{\aleph_{2}}^{\aleph_{1}}}$; see King-Steinhorn $[\mathrm{Ki}-\mathrm{St}]$.

\section{REFERENCES}

[Ba-Ta] J. Baumgartner and A. Taylor, Saturation properties of ideals in generic extensions I, II, Trans. Am. Math Soc. 270 (1982), 557-574 and 271 (1982), 587-609. MR 83k:03040a,b

[Ba-Ta-Wa] J. Baumgartner, A. Taylor and S. Wagon, On splitting stationary subsets of large cardinals, J. Sym Logic 42 (1977), 203-214. MR 58:21619

[Br-Ma] M. Burke and M. Magidor, Shelah's pcf theory and its applications, Ann. Pure and Ap. Logic 50 (1990), 207-254. MR 92f:03053

[Cu-Wo] J. Cummings and H. Woodin, Generalized Prikry Forcing (to appear).

[Dz-Sh] M. Džamonja and S. Shelah, On squares, outside guessing of clubs and $I_{<f}[\lambda]$, Fund. Math. 148 (1995), 165-198. CMP 96:04.

[Dz-Sh2] M. Džamonja and S. Shelah, Saturated filters at filters at successors of singulars, weak reflection (to appear; Shelah's paper 545).

[Fo] M. Foreman, More saturated ideals, Cabal Seminar 79-81,Lecture Notes in Math. 1019, Springer, 1983, pp. 1-27. MR 86j:03002

[Fo-Ma-Sh] M. Foreman, M. Magidor and S. Shelah, Martin's Maximum, saturated ideals and nonregular ultrafilters, I, Ann. of Math. 127 (1988), 1-47. MR 89f:03043

[Fo-Wo] M. Foreman and H. Woodin, GCH can fail everywhere, Ann. of Math. 133 (1991), 1-36. MR 91k:03130

[Gi1] M. Gitik, The nonstationary ideal on $\aleph_{2}$, Israel Journal of Math. (1984), 257-288. MR 86i:03061

[Gi2] M. Gitik, Changing cofinalities and the nonstationary ideal, Israel Journal of Math. 56 (1986), 280-314. MR 89b:03086

[Gi3] M. Gitik, Some results on the nonstationary ideal, Israel Journal of Math. 92 (1995), 61-112. CMP 96:03

[Gi4] M. Gitik, Some results on nonstationary ideal 2, to appear, Israel J. of Math.

[Gr] J. Gregory, Higher Souslin trees and GCH, J. Symb. Logic 41 (1976), 663-671. MR 58:5208

[Je-Wo] T. Jech and H. Woodin, Saturation of the closed unbounded filter on the set of regular cardinals, Trans. Am. Math. Soc. 292 (1) (1985), 345-356. MR 87d:03139

[Je-Ma-Mi-Pr] T. Jech, M. Magidor, W. Mitchell and K. Prikry, Precipitous ideals, J. Symb. Logic 45 (1980), 1-8. MR 81h:03097

[Je-Pr] T. Jech and K. Prikry, Ideals of sets and the power set operation, Bul. Am. Math. Soc. 82 (1976), 593-595. MR 58:21618 
[Ki-St] J. King and C. Steinhorn, The uniformization property for $R_{2}^{\prime}$, Israel J. Math. 36 (1980), 248-256. MR 82a:03051

$[\mathrm{Ku}] \quad$ K. Kunen, Saturated ideals, J. of Sym. Logic 43 (1978), 65-76. MR 80a:03068

[Na] K. Namba, On closed unbounded ideal of ordinal numbers, Comm. Univ. Sancti Pauli 22 (1974), 33-56. MR 50:4308

[Sh1] S. Shelah, Proper forcing, Lec. Notes in Math. 940, Springer-Verlag, 1982. MR 84h:03002

[Sh2] S. Shelah, Cardinal Arithmetic, Oxford Univ. Press, 1994. MR 96e:03001

[Sh3] S. Shelah, Appendix: on stationary sets (to "Classification of nonelementary classes II). Abstract elementary classes") in Proc. of the USA-Israel Conference on Classification theory, Lec. Notes in Math. 1292 (J. Baldwin, ed.), Springer, 1987, pp. 483-485. MR 91h:03046

[Sh4] S. Shelah, On Successors of Singular Cardinals, in Logic Colloquium 78 (M. Boffa, D. van Dalen and K. McAloon, eds.), North Holland, 1979, pp. 357-380. MR 82d:03079

[Sh5] S. Shelah, Diamonds and uniformizations, J. Symb. Logic 49 (1984), 1022-1033. MR 86g:03083

[Sh6] S. Shelah, Iterated forcing and normal ideals on $\omega_{1}$, Is. Jour. of Math. 60 (1987), 345-380. MR 90g:03050

[Sh7] S. Shelah, More on stationary coding, in Around Classification Theory of Models, Lecture Notes in Math. 1182 (1986), 224-246. MR 90a:03037

[Sh8] S. Shelah, Non structure theory, Oxford Univ. Press ([Sh-h]) (to appear).

[Sh9] S. Shelah, The Generalized Continuum Hypothesis Revisited ([Sh460]) . (to appear).

[So] R. Solovay, Real valued measurable cardinals, in Axiomatic Set Theory, Proceedings of Sym. in Pure Math. XIII 1 (1971), 387-428. MR 45:55

[St] J. Steel, The Core Model Iterability Problem (to appear).

[St-V] J. Steel and R. Van Wesep, Two consequence of determinacy consistent with choice, Trans. An. Math. Soc. 272 (1) (1982), 67-85. MR 83i:03081

[Wo1] H. Woodin, Some consistency results yin $Z F$ using AD, Cabal Seminar, Lect. Notes in Math. 1019, Springer, 1983, pp. 172-199. MR 86j:03002

School of Mathematical Sciences, Sackler Faculty of Exact Sciences, Tel Aviv UniVersity, Ramat Aviv 69978, IsRael

E-mail address: gitik@math.tau.ac.il

Hebrew University of Jerusalem, Department of Mathematics, Givat Ram, Jerusalem, ISRAEL

E-mail address: shelah@math.huji.ac.il 